\title{
ON A PROBLEM OF CHEVALLEY
}

\author{
KATSUHIKO MASUDA
}

Recently Prof. Chevalley in Nagoya suggested to the author the following problem: Let $k$ be a field, $K_{5}=k\left(x_{1}, x_{2}, x_{3}, x_{4}, x_{5}\right)$ be a purely transcendental extension field (of transcendental degree 5) of $k, s_{5}$ be the cyclic permutation of $x: s_{5} x_{1}=x_{2} s_{5} x_{2}=x_{3} s_{5} x_{3}=x_{4} s_{5} x_{4}=x_{5} s_{5} x_{5}=x_{1}$, and let $L_{5}$ be the field of invariants of $s_{5}$ in $K_{5}$. Is $L_{5}$ then purely transcendental over $k$ or not? When the characteristic $p$ of $k$ is not equal to 5 , it is answered in the following positively. When the characteristic $p$ of $k$ is equal to 5 , it is answered also positively by Mr. Kuniyoshi's result in [2].

Now let $K_{n}=k\left(x_{1}, x_{2}, x_{3}, \ldots, x_{n}\right)$ be a purely transcendental extension field (of transcendental degree $n$ ) of $k, s_{n}$ be the cyclic permutation of $x: s_{n} x_{1}$ $=x_{2} s_{n} x_{2}=x_{3} \ldots s_{n} x_{n}=x_{1}$, and let $L_{n}$ be the field of invariants of $s_{n}$ in $K_{n}$. We suppose from now on throghout the present article that $n$ is not divisible by the characteristic $p$ of $k$. If the ground field $k$ involves a primitive $n$-th root $\zeta_{n}$ of 1 , we can see easily that $L_{n}$ is purely transcendental over $k$. From this fact we obtain in the following that existence of certain sets of primitive generators of $L_{n}\left(\zeta_{n}\right)$ over $k\left(\zeta_{n}\right)$ (the definition is shown in the following) is a necessary and sufficient condition for $L_{n}$ to be purely transcendental over $k$, and the existence of such sets of primitive generators are shown for every case of $n \leqq 7$ through calculations on factor sets ${ }^{1}$. It looks that a more arithmetical approach will be necessary to solve the problem with reference to general $n$.

1. Let $k_{n}^{\prime}=k\left(\zeta_{n}\right), K_{n}^{\prime}=K_{n}\left(\zeta_{n}\right)$ and $\&$ be the Galois group of $k^{\prime}$ over $k$. We omit all $n$ as subscripts throughout in the following, unless indispensable. Let $L^{\prime}$ denote the field of invariants of $s$ in $K^{\prime} . \quad K$ and $K^{\prime}$ are clearly Galois extension fields over $L$ and $L^{\prime}$ of the same rank $n$ respectively. Their Galois groups are generated by the automorphism induced by $s$. We do not distin-

Received June 1, 1954.

1) Cf. [1] and [3]. Hasse factor sets defined in [3] without the supposition that the absolutely irreducible representations of Galois groups are obtained in the ground field has close relations to the problem of the pure transcendency of $L_{n}$ over $k$. 
guish these two Galois groups and the cyclic permutation group of $x$ generated by $s$ and denote them by same $\mathbb{B}$. As $K$ is purely transcendental over $k, K$ and $k^{\prime}$ are linearly disjoint over $k^{2)}$ and $\left[K^{\prime}: K\right]=[L(\zeta): L]=\left[k^{\prime}: k\right]$. The restrictions of the Galois group of $K^{\prime}$ over $K$ into $L(\zeta)$ and $k^{\prime}$ are the Galois group of $L(\zeta)$ over $L$ and the Galois group of $k^{\prime}$ over $k$ respectively. We do not distinguish these three Galois groups and denote them by same $\mathbb{B}$. Then we can see easily from the Galois theory that $L(\zeta) \cap K=L$ and $[K: L]$ $=\left[K^{\prime}: L(\zeta)\right]=\left[K^{\prime}: L^{\prime}\right]$. As $L(\zeta) \subset L^{\prime}$, we obtain now the following Lemma.

LEMMA 1. $L^{\prime}=L(\zeta), L^{\prime} \cap K=L$ and $\left[L^{\prime}: L\right]=\left[k^{\prime}: k\right]$.

Let $y_{j}=\sum_{i=1}^{n} \zeta^{-i j} x_{i}$ and $c_{j, k}=y_{j} y_{k} / y_{j+k}$ for $j, k=1,2, \ldots, n$, where we denote by $\overline{j+k}$ the integer determined uniquely by $\overline{j+k} \equiv j+k \bmod n$ and $1 \leqq \overline{j+k}$ $\leqq n$. $\quad c_{j, k}$ belongs clearly to $L^{\prime}$. Let $M^{\prime}$ denote the field generated over $k^{\prime}$ by all $c_{j, k}$ for $j, k=1,2, \ldots, n$. From $c_{i, j}=c_{1, j} c_{1, \pi}, \ldots c_{1, j+i} / c_{1,1} c_{1,2} \ldots c_{1, i-1}$ it follows easily that $M^{\prime}=k^{\prime}\left(c_{1,1} c_{1,2}, \ldots, c_{1, n}\right)$ and $y^{n} \in M^{\prime}$. As $y_{1}$ gives an isomorphic irreducible representation of $\mathbb{B},\left[M^{\prime}\left(y_{1}\right): M^{\prime}\right]=n$. As $y_{2}, y_{3}, \ldots$, $y_{n}$ can be written as rational combinations of $y_{1}$ and $c_{j, k}$ over $M^{\prime}$ with coefficients in $k^{\prime}, M^{\prime}\left(y_{1}\right)=M^{\prime}\left(y_{1}, y_{2}, \ldots, y_{n}\right)=K^{\prime}$. So $\left[K^{\prime}: M^{\prime}\right]=n, M^{\prime}=L^{\prime}$ and $L^{\prime}=k^{\prime}\left(c_{1,1}, c_{1,2}, c_{1,3}, \ldots, c_{1, n}\right)$. As the transcendental degree of $L^{\prime}$ is $n$, we obtain

THEOREM 1. $L^{\prime}$ is purely transcendental over $k^{\prime}$.

We call a set $\left(a_{1}, a_{2}, \ldots, a_{t}\right)$ of elements in $L^{\prime}$ a primitive generating set of $L^{\prime}$ over $k(\zeta)$, if $\sum_{i=1}^{t} \iota\left(a_{i}\right)=n$ and $L^{\prime}=k^{\prime}\left(a_{1}, a_{1}^{\prime}, a_{1}^{\prime \prime}, \ldots a_{1}^{\left(\ell\left(a_{1}\right)-1\right)}, a_{2}, a_{2}^{\prime}, a_{2}^{\prime \prime}, \ldots\right.$, $\left.a_{2}^{\left(\ell\left(a_{2}\right)-1\right)}, \ldots, a_{t}, a_{t}^{\prime}, a_{t}^{\prime \prime}, \ldots, a_{t}^{\left(\ell\left(a_{t}\right)-1\right)}\right)$, where we denote by $\iota^{(}\left(a_{i}\right)$ the number of (different) conjugate elements of $a_{i}$ over $L$. So the number $t$ of elements in such a set is not greater than $n, c\left(a_{i}\right)=\left[L\left(a_{i}\right): L\right]$ and $a_{i}^{(j)} \neq a_{i^{\prime}}^{\left(j^{\prime}\right)}$ except only when $i=i^{\prime}, j=j^{\prime}$. As $\&$ is an abelian group, $L\left(a_{i}\right)$ is a Galois extension field of $L$ and $L\left(a_{i}, a_{i}^{\prime}, a_{i}^{\prime \prime}, \ldots, a_{i}^{\left(\ell\left(a_{i}\right)-1\right)}\right)=L\left(a_{i}\right)$. Now we prove the following theorem.

THEOREM 2. $L$ is purely transcendental over $k$, if and only if there exists a primitive generating set of $L(\zeta)$ over $k(\zeta)$.

Proof. (i) Sufficiency. Let $\left(a_{1}, a_{2}, \ldots, a_{t}\right)$ be a primitive generating set

2) Cf. Chap. I. $\$ 7$ in [4]. 
of $L^{\prime}$ over $k$. Let $k_{i}^{\prime}=L\left(a_{i}\right) \cap k^{\prime}$ for $i=1,2, \ldots, t$. Then $L\left(a_{i}\right)=L k_{i}^{\prime}$ and the Galois group of $L\left(a_{i}\right)$ over $L$ is equal to the Galois group of $k_{i}^{\prime}$ over $k$. Let $\omega_{i, 1}, \omega_{i, 2}, \ldots, \omega_{i, \iota a_{i}}$ be a normal basis of $k^{\prime}$ over $k$ (accordingly also such one of $L\left(a_{i}\right)$ over $\left.L\right)$. $a_{i}$ can be written as $a_{i}=\sum_{j=1}^{l\left(a_{i}\right)} \omega_{i, j} m_{j, i}$ with $m_{j, i}$ in $L$ for $i=1$, $2, \ldots, t . a_{i}, a_{i}^{\prime}, a_{i}^{\prime \prime}, \ldots, a_{i}^{\ell\left(a_{i}\right)-1}$ are clearly written as bilinear combinations of $\omega_{i, 1}, \omega_{i, 2}, \ldots, \omega_{i, \iota\left(a_{i}\right)}$ and $m_{1, i}, m_{2, i}, \ldots, m_{\iota\left(a_{i}\right), i}$. As $a_{i}, a_{i}^{\prime}, a_{i}^{\prime \prime}, \ldots, a_{i}^{\left(\ell\left(a_{i}\right)-1\right)}$ are algebraically independent over $k^{\prime}$, these forms are as linear combinations of $m_{1, i}, m_{2, i}, \ldots, m_{\iota\left(a_{i}\right), i}$ with coefficients in $k^{\prime}$ linearly independent. So $m_{1, i}, m_{2, i}$, $\ldots, m_{\ell\left(a_{i}\right), i}$ can be written as linear combinations of $a_{i}, a_{i}^{\prime}, a_{i}^{\prime \prime}, \ldots, a_{i}^{\left(\ell\left(a_{i}\right)-1\right)}$ with coefficients in $k_{i}^{\prime}$ and so $k^{\prime}\left(a, a_{i}^{\prime}, a_{i}^{\prime \prime}, \ldots, a_{i}^{\left(\ell\left(a_{i}\right)-1\right)}\right)=k^{\prime}\left(m_{1, i}, m_{2, i}, \ldots, m_{\ell\left(a_{i}\right), i}\right)$. Thus we obtain $L^{\prime}=k^{\prime}\left(a_{1}, a_{1}^{\prime}, a_{1}^{\prime \prime}, \ldots, a_{1}^{\left(\ell\left(a_{1}\right)-1\right)}, a_{2}, a_{2}^{\prime}, a_{2}^{\prime \prime}, \ldots, a_{2}^{\left(\ell\left(a_{2}\right)-1\right)}, \ldots\right.$, $\left.a_{t}, a_{t}^{\prime}, a_{t}^{\prime \prime}, \ldots, a_{t}^{\left(\ell\left(a_{t}\right)-1\right)}\right)=k^{\prime}\left(m_{1,1}, m_{2,1}, \ldots, m_{\iota\left(a_{1}\right), 1}, m_{1,2}, m_{2,2}, \ldots, m_{\iota\left(a_{2}\right), 2},\right.$. $\left.\ldots, m_{1, t}, m_{2, t}, \ldots, m_{\ell\left(a_{t}\right), t}\right)$. Let $M=k\left(m_{1,1}, m_{2,1}, \ldots, m_{\iota\left(a_{1}\right), 1}, m_{1,2}, m_{2,2}, \ldots\right.$ $\left.., m_{\ell\left(a_{2}\right), 2}, \ldots, m_{1, t}, m_{2, t}, \ldots, m_{\ell\left(a_{t}\right) t}\right)$. Then $M \cong L$. As $M k^{\prime}=L^{\prime}, L^{\prime}$ is algebraic over $M$ and $\left[L^{\prime}: M\right] \leqq\left[k^{\prime}: k\right]$, so $M=L$. As the transcendental degree of $L(=M)$ is $n, m$ 's are algebraically independent generators of $L$ and $L$ is purely transcendental over $k$.

(ii) Necessity. Suppose that $L$ is purely transcendental over $k$ and $L=k\left(a_{1}\right.$, $\left.a_{2}, \ldots, a_{n}\right) . \quad\left(a_{1}, a_{2}, \ldots, a_{n}\right)$ is clearly a primitive generating set of $L^{\prime}$ over $k(\zeta)$. q.e.d.

2. Now we prove the following theorem.

TheOREM 3. Let $n \leqq 7$ and suppose that the characteristic $p$ of $k$ does not divide $n$. Then $L$ is purely transcendental over $k$.

Proof. (i) When $n=1$, the theorem is trivial.

(ii) When $n=2$, it holds $\left[k^{\prime}: k\right]=1$ from $p+n$ and the theorem follows from Theorem 1.

(iii) When $n=3,\left[k^{\prime}: k\right]=1$ or 2. If $\left[k^{\prime}: k\right]=1$, the theorem follows from Theorem 1. If $\left[k^{\prime}: k\right]=2$, let $a_{1}=c_{1,3}=x_{1}+x_{2}+x_{3}$ and $a_{2}=c_{1,1}$ $=\left(\zeta_{3} x_{1}+\zeta_{3}^{2} x_{2}+x_{3}\right)^{2} / \zeta_{3}^{2} x_{1}+\zeta_{3} x_{2}+x_{3}$. Then $\iota\left(a_{1}\right)+\iota\left(a_{2}\right)=1+2=3$ and since $a_{2}$ $=c_{2,2}=c_{1,2} c_{1,3} / c_{1,1}$ it follows $k^{\prime}\left(a_{1}, a_{2}, a_{2}^{\prime}\right)=k^{\prime}\left(c_{1,1}, c_{1,2}, c_{1,3}\right)=L_{3}^{\prime}$. So $\left(a_{1}, a_{2}\right)$ is a primitive generating set of $L_{3}^{\prime}$ over $k\left(\zeta_{3}\right)$ and the theorem follows from Theorem 2.

(iv) When $n=4,\left[k^{\prime}: k\right]=1$ or 2 . If $\left[k^{\prime}: k\right]=1$, the theorem fol- 
lows from Theorem 1. When $\left[k^{\prime}: k\right]=2$, let $a_{1}=c_{1,4}, a_{2}=c_{1,2}, a_{3}=c_{1,3}$. Then $\imath\left(a_{1}\right)+\imath\left(a_{2}\right)+\imath\left(a_{3}\right)=1+2+1$ and since $a_{2}^{\prime}=c_{3,2}=c_{1,3} c_{1,4} / c_{1,1}$ it follows $k^{\prime}\left(a_{1}\right.$, $\left.a_{2}, a_{2}^{\prime}, a_{3}\right)=k^{\prime}\left(c_{1,1}, c_{1,2}, c_{1,3}, c_{1,4}\right)=L_{4}^{\prime}$. So $\left(a_{1}, a_{2}, a_{3}\right)$ is a primitive generating set of $L_{4}^{\prime}$ over $k\left(\zeta_{1}\right)$ and the theorem follows from Theorem 2.

(v) When $n=5,\left[k^{\prime}: k\right]=1$ or 2 or 4 . If $\left[k^{\prime}: k\right]=1$, the theorem follows from Theorem 1 . When $\left[k^{\prime}: k\right]=4$, let $a_{1}=c_{1,5}, a_{2}=c_{1,2}$. Then $\left.c^{(} a_{1}\right)$ $+\iota\left(a_{2}\right)=1+4=5$ and it follows from $a_{2}^{\prime}=c_{2,4}=c_{1,4} c_{1,5} / c_{1,1}, a_{2}^{\prime \prime}=c_{1,3}, a_{2}^{\prime \prime \prime}=c_{\hat{n}, 4}$ $=c_{1,1} c_{1,5} / c_{1,2}$ that $k^{\prime}\left(a_{1}, a_{2}, a_{2}^{\prime}, a_{2}^{\prime \prime}, a_{2}^{\prime \prime \prime}\right)=k^{\prime}\left(c_{1,1}, c_{1,2}, c_{1,3}, c_{1,4}, c_{1,5}\right)=L_{5}^{\prime}$. So $\left(a_{1}\right.$, $\left.a_{2}\right)$ is a primitive generating set of $L_{\overline{5}}^{\prime}$ over $k\left(\zeta_{\overline{5}}\right)$, and the theorem follows from Theorem 2. If $\left[k^{\prime}: k\right]=2$ it is easily seen that one of the following three sets $\left(c_{1,5}, c_{1,2}, c_{2,1}\right),\left(c_{1,5}, c_{1,2}, c_{1,3}\right),\left(c_{1,5}, c_{1,2}, c_{3,1}\right)$ becomes a primitive generating set of $L\left(\zeta_{5}\right)$ over $k\left(\zeta_{5}\right)$.

(vi) When $n=6,\left[k^{\prime}: k\right]=1$ or 2 . When $\left[k^{\prime}: k\right]=1$, the theorem follows from Theorem 1. When $\left[k^{\prime}: k\right]=2$, let $a_{1}=c_{1,6}, a_{2}=c_{1,2}, a_{3}=c_{1,4}, a_{4}=c_{1,5}$, then $c\left(a_{1}\right)+\imath\left(a_{2}\right)+\iota\left(a_{3}\right)+\iota\left(a_{4}\right)=1+2+2+1=6$, and it follows from $a_{2}^{\prime}=c_{5,4}$ $=c_{1,5}, c_{1,6} / c_{1,3}, a_{3}^{\prime}=c_{5,2}=c_{1,5}, c_{1,6} / c_{1,1}$ that $k^{\prime}\left(a_{1}, a_{2}, a_{2}^{\prime}, a_{3}, a_{3}^{\prime}, a_{4}\right)=k^{\prime}\left(c_{1,1}, c_{1,2}\right.$, $\left.c_{1,3}, c_{1,4}, c_{1,5}, c_{1,6}\right)=L_{6}^{\prime}$. So $\left(a_{1}, a_{2}, a_{3}, a_{4}\right)$ is a primitive generating set of $L_{6}^{\prime}$ over $k\left(\zeta_{6}\right)\left(=k^{\prime}\left(\zeta_{3}\right)\right)$ and the theorem follow's from Theorem 2 .

(vii) When $n=7,\left[k^{\prime} ; k\right]=1$ or 2 or 3 or 6 . If $\left[k^{\prime}: k\right]=1$, the theorem follows from Theorem 1. In the case of $\left[k^{\prime}: k\right]=6$, let $a_{1}=c_{1, \tau}, a_{2}$ $=c_{1,3}$. Then $6\left(a_{1}\right)+\iota^{2}\left(a_{2}\right)=1+6=7$, and $a_{2}^{\prime}=c_{2,3}, a_{2}^{\prime \prime}=c_{2,6}, a_{2}^{\prime \prime \prime}=c_{4,6}, a_{2}^{\prime \prime \prime \prime}=c_{4,5}$, $\boldsymbol{a}_{2}^{\prime \prime \prime \prime \prime}=c_{1,5}$. It follows from $c_{1,2}=c_{4,6}, c_{1,5} / c_{4,5}, c_{1,4}=c_{2,3}, c_{4,6} / c_{2,6}, c_{1,1}=c_{2,6} / c_{4,6}$, $c_{1,3}$ that $k^{\prime}\left(a_{1}, a_{2}, a_{2}^{\prime}, a_{2}^{\prime \prime}, a_{2}^{\prime \prime \prime}, a_{2}^{\prime \prime \prime}, a_{2}^{\prime \prime \prime \prime \prime}\right)=k^{\prime}\left(c_{1,1}, c_{1,2}, c_{1,3}, c_{1,4}, c_{1,5}, c_{1,6}, c_{1,7}\right)$ $=L^{\prime}$, and $\left(a_{1}, a_{2}\right)$ is a primitive generating set of $L_{i}^{\prime}$ over $k\left(\zeta_{i}\right)$ and the theorem follows from Theorem 2. If $\left[k^{\prime}: k\right]=3$ or 2 , we can find easily a primitive generating set in $\left(c_{1, i}, c_{1,3}, c_{2,3}, c_{2,6}, c_{4,6}, c_{4,5}, c_{1,5}\right)$ and the theorem follows from Theorem 2 .

In the following we give polynomials of $x$ which are algebraically independent generators of $L_{n}$ over $k$ for $n \leqq 4$ obtained easily from the above primitive generators.

When $n=1 ; \quad x_{1}$.

$$
\begin{aligned}
n=2 ; \quad x_{1}+x_{2}, x_{1} x_{2} . & \\
n=3 ; \quad x_{1}+x_{2}+x_{3}, & \left(\sum_{i=1}^{3} x_{i} x^{2} \overline{i+1}-x_{1} x_{2} x_{3}\right) /\left(\sum_{i=1}^{3} x_{i}^{2}-\sum_{i=1}^{3} x_{i} x_{i+1}\right), \\
& \left(\sum_{i=1}^{3} x_{i} x_{i+1}^{2}-x_{1} x_{2} x_{3}\right) /\left(\sum_{i=1}^{3} x_{i}^{2}-\sum_{i=1}^{3} x_{i} x_{i+1}\right) .
\end{aligned}
$$




$$
\begin{aligned}
n=4 ; \quad x_{1}+ & x_{2}+x_{3}+x_{1}, \sum_{i=1}^{4} x_{i}^{2}-2\left(x_{1} x_{3}+x_{2} x_{1}\right) \\
& \sum_{i=1}^{4} x_{i}^{3}-\sum_{i \neq j} x_{i}^{2} x_{j}+2 x_{1} x_{2} x_{3} x_{i},-\sum_{i=1}^{4} x_{i}^{2} x_{i+1}+\sum_{i=1}^{4} x_{i}^{2} x_{i+3} .
\end{aligned}
$$

This shows that $L_{n} \cap k\left[x_{1}, x_{2}, \ldots, x_{n}\right]$ is purely transcendental integral domain over $k$, when $n=1,2,4$.

\section{REFERENCES}

[1] H. Hasse : Invariante Kennzeichnung Galoisschen Körper mit vorgegebener Galoisgruppe, Crelle J., 187 (1949).

[2] H. Kuniyoshi : On a problem of Chevalley, the present volume of this Journal.

[3] K. Masuda : One valued mappings of groups into fields, Nagoya Math. J., 6 (1953).

[4] A. Weil: Foundations of Algebraic Geometry, New York (1946).

Department of Mathematics

Yamagata University 